\title{
Modelagem e propriedades termodinâmicas na secagem de morangos
}

\author{
Modelling and thermodynamic properties of the drying of strawberries
}

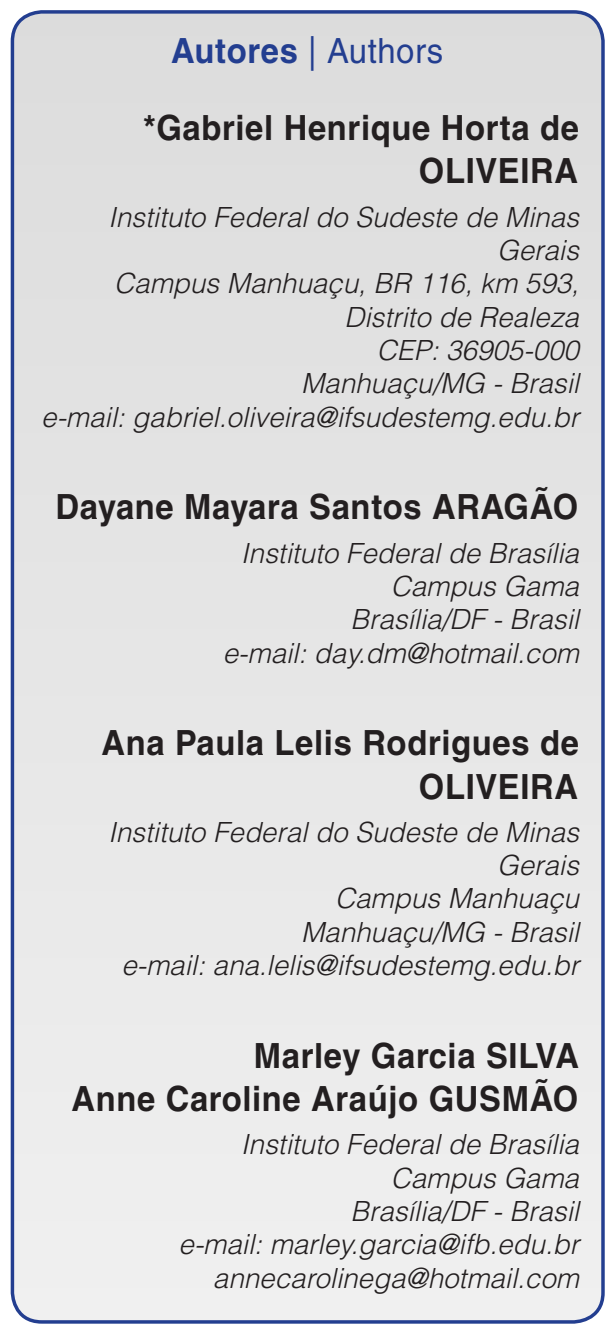

*Autor Correspondente / Corresponding Author

Recebido: Jul. 02, 2015

Aprovado: Dez. 08, 2015

\section{Resumo}

A secagem de produtos agrícolas é largamente utilizada no mundo para diminuir o teor de água do produto, dificultando o crescimento microbiano e a ocorrência de reações químicas e bioquímicas e possibilitando a armazenagem segura por períodos longos, além de reduzir o volume e a massa a ser transportada. O objetivo do presente trabalho foi avaliar e modelar a etapa de secagem de morangos (Fragaria L.), bem como obter parâmetros termodinâmicos em três diferentes temperaturas $\left(40^{\circ} \mathrm{C}, 50^{\circ} \mathrm{C}\right.$ e $\left.60^{\circ} \mathrm{C}\right)$. Foram utilizados frutos obtidos na CEASA-DF, com teor de água inicial de 93,0\% (b.u.). Os dados experimentais foram ajustados a seis modelos matemáticos usualmente utilizados para a representação da etapa de secagem. A energia de ativação para a secagem dos morangos, a entropia, a entalpia e a energia livre de Gibbs foram obtidas. O modelo Logaritmo foi o que melhor representou a secagem de frutos de morangueiro, apresentando valores de erro médio relativo entre $7,59 \%$ e 8,48\%, desvio-padrão da estimativa entre 0,03 e 0,06 e coeficiente de determinação entre $99,93 \%$ e $99,95 \%$. A entalpia do processo de secagem diminuiu com o aumento da temperatura de secagem, com valores de $-481,21,-564,35$ e $-647,49 \mathrm{~J} \mathrm{~mol}^{-1}$ para as temperaturas 40,50 e $60^{\circ} \mathrm{C}$, respectivamente. O mesmo comportamento ocorreu com a entropia, com valores $-238,35,-238,61$ e $-238,86 \mathrm{~J} \mathrm{~mol}^{-1} \mathrm{~K}^{-1}$, para temperaturas 40,50 e $60^{\circ} \mathrm{C}$, respectivamente. A energia livre de Gibbs aumentou com o aumento da temperatura, atingindo valores de 74.159,75, 76.544,54 e $78.931,90 \mathrm{~J} \mathrm{~mol}^{-1}$ para as temperaturas 40,50 e $60{ }^{\circ} \mathrm{C}$, respectivamente.

Palavras-chave: Teor de água; Entalpia; Entropia; Energia livre de Gibbs.

\section{Summary}

The drying of agricultural products is widely used throughout the world to diminish the moisture content of the product, making microbial growth and chemical and biochemical reactions difficult, enabling safe storage for longer periods and also reducing the volume and mass to be transported. The aim of the present work was to evaluate and model the drying process of strawberries (Fragaria L.) and obtain the thermodynamic parameters at three different temperatures $\left(40,50\right.$ and $\left.60^{\circ} \mathrm{C}\right)$. Fruits purchased at CEASA-DF with an initial moisture content of $93 \%$ (w.b.) were used. The experimental data were fitted to six mathematical models commonly employed to represent the drying step. The activation energy for the drying of strawberries and also the entropy, enthalpy and Gibbs free energy were determined. The Logarithmic model was the one that best fitted the drying phenomenon of strawberry fruits, presenting values for the mean relative error between 7.59 and $8.48 \%$, with an estimated standard error between 0.03 and 0.06 and determination coefficient between 99.93 and $99.95 \%$. The enthalpy of the drying process decreased with increase in drying temperature, with values of $-481.21,-564.35$ and $-647.49 \mathrm{~J} \mathrm{~mol}^{-1}$ for temperatures of 40,50 and $60^{\circ} \mathrm{C}$, respectively. The same trend occurred for entropy, with values of $-238.35,-238.61$ and $-238.86 \mathrm{~J} \mathrm{~mol}^{-1} \mathrm{~K}^{-1}$ for the temperatures of 40,50 and $60^{\circ} \mathrm{C}$, respectively. Gibbs free energy increased with increase in temperature, attaining values of $74,159.75,76,544.54$ and $78,931.90 \mathrm{~J} \mathrm{~mol}^{-1}$ for the temperatures of 40,50 and $60^{\circ} \mathrm{C}$, respectively.

Key words: Moisture content; Enthalpy; Entropy; Gibbs free energy. 


\section{Introdução}

O morangueiro (Fragaria L.) é cultivado em todos os continentes, com intensidade nos Estados Unidos, Espanha, Japão, Itália, Coréia do Sul e Polônia (REISSER JUNIOR et al., 2010). No Brasil, a cultura encontra-se difundida em regiões de clima temperado e subtropical, nas quais se produz morango para consumo in natura e para a industrialização, sendo os estados de São Paulo, Minas Gerais e Rio Grande do Sul os maiores produtores.

O cultivo do morango também se adapta a regiões quentes, como o cerrado (ANTUNES; REISSER JUNIOR, 2007). No Distrito Federal, ele ocupa em torno de 200 hectares e no período de safra a produção chega a 6,7 toneladas/ano, sendo a principal fonte de renda agrícola da região administrativa de Brazlândia (EMATER, 2013). A produção de morangos expande-se a cada ano, predominando o cultivo em pequenas propriedades rurais, com mão de obra familiar (RESENDE et al., 1999).

A grande popularidade desse fruto deve-se à coloração, ao aroma e ao sabor, assim como a suas propriedades nutricionais, que fazem do morango um produto muito apreciado pelos consumidores. Por ser um produto de alta perecibilidade in natura, o morango requer a utilização de tecnologias de pós-colheita adequadas para sua melhor conservação e ampliação da vida de prateleira, sendo também processado na indústria para agregar valor e obter novos produtos. Os morangos são transformados em: balas, sucos, sorvetes, geleias e iogurtes. Essas transformações requerem tecnologias adequadas para possibilitar o correto manuseio na fabricação.

Dentre as tecnologias, a secagem é a mais conhecida e utilizada no mundo para assegurar a qualidade de produtos in natura e a estabilidade durante a vida de prateleira dos mesmos. A diminuição da quantidade de água (teor de água) do material pela secagem reduz a atividade biológica e as mudanças químicas e físicas durante os procedimentos pós-colheita até o consumo final. Consiste procedimento imprescindível para a transformação industrial de frutas em subprodutos.

A conservação pela secagem baseia-se no fato de que tanto os microrganismos como as enzimas e todo o mecanismo metabólico necessitam de água para suas atividades. Com a redução da quantidade de água disponível, serão reduzidas a atividade de água e a velocidade das reações químicas e, como consequência, o desenvolvimento de microrganismos, conferindo ao produto uma maior qualidade por maior período de tempo, aumentando-se, assim, a vida de prateleira.

Diferentes trabalhos envolvendo a secagem do morango foram realizados nos últimos anos envolvendo secagem osmótica (NUÑEZ-MANCILLA et al., 2011;
2013), secagem a vácuo (MORENO et al., 2012), secagem por micro-ondas (CONTRERAS et al., 2008), secagem usando energia solar (EL-BELTAGY et al., 2007) e secagem por liofilização (MOSQUERA et al., 2012). Sousa et al. (2014) estudaram a cinética de secagem de morangos cortados longitudinalmente a $40{ }^{\circ} \mathrm{C}$, $55^{\circ} \mathrm{C}$ e $70{ }^{\circ} \mathrm{C}$. Entretanto, a secagem dos morangos em sua forma íntegra não foi realizada, uma vez que alterações na forma e tamanho dos frutos incorrem em alterações na cinética de secagem, consequentemente alterando o dimensionamento e a correta secagem do produto. As propriedades termodinâmicas da secagem, necessárias para o cálculo da energia necessária para que ocorra o processo, também não foram obtidas. O estudo da secagem convencional aplicada ao morango em diferentes temperaturas é relevante, devido à facilidade de acesso e uso dessa tecnologia pelos produtores e profissionais ligados à cadeia produtiva do morango.

A simulação do comportamento de cada alimento durante a redução do teor de água é importante no desenvolvimento e aprimoramento de equipamentos de secagem, e para tal utilizam-se modelos matemáticos que possam representar satisfatoriamente a perda de água durante o período de secagem em função da espessura de camadas do material (BERBERT et al., 1995). Além da simulação, o conhecimento das propriedades termodinâmicas nas etapas de secagem de produtos agrícolas também é importante fonte de informação para projetar secadores, calcular a energia requerida na etapa, estudar as propriedades da água adsorvida, avaliar a microestrutura dos alimentos e estudar os fenômenos físicos que ocorrem na superfície dos alimentos (OLIVEIRA et al., 2011).

Mudanças de entalpia fornecem uma medida da variação de energia que ocorre na interação das moléculas de água com os constituintes do produto durante os processos de sorção. A entropia pode estar associada com a ligação ou repulsão das moléculas de água dos componentes do alimento no sistema e está associada com o arranjo espacial da relação água-produto. Assim, de acordo com Oliveira et al. (2011), a entropia caracteriza, ou define, o grau de ordem ou desordem existente no sistema água-produto. Já a energia livre de Gibbs é um indicativo da afinidade do produto com a água, fornecendo um critério de avaliação da dessorção de água. Mudanças na energia livre de Gibbs durante a troca de água entre o produto e a vizinhança são associadas à energia requerida para transferir moléculas de água do estado vapor para uma superfície sólida ou vice-versa.

Considerando-se a importância do estudo teórico na etapa de secagem dos produtos agrícolas, o objetivo do 
trabalho foi obter as curvas de secagem nas temperaturas $40^{\circ} \mathrm{C}, 50^{\circ} \mathrm{C}$ e $60^{\circ} \mathrm{C}$ de morangos, efetuando-se ajustes a modelos matemáticos, bem como obter as propriedades termodinâmicas durante a desidratação.

\section{Material e métodos}

O trabalho foi realizado no Laboratório de Qualidade e Propriedades Físicas e Químicas de Produtos Vegetais do Instituto Federal de Educação, Ciência e Tecnologia de Brasília, campus Gama.

Foram utilizados frutos de morangueiro (Fragaria L.) adquiridos na CEASA-DF com teor de água inicial de $0,93 \mathrm{~kg}$ água $/ \mathrm{kg}$ de morango (b.u.). Para a determinação do teor de água, foi utilizado o método padrão de estufa $\left(105 \pm 3{ }^{\circ} \mathrm{C}\right.$, durante $24 \mathrm{~h}$ ), de acordo com as Regras para Análise de Sementes (BRASIL, 1992), até massa constante. As análises foram realizadas em triplicata.

Previamente à secagem, os morangos foram submetidos a limpeza, utilizando-se água corrente. Não foram retiradas quaisquer outras partes dos frutos, tais como pedicelos e sépalas. Para a secagem, as amostras foram preparadas baseando-se em uma proporção de massa de produto/área da bandeja de $1,5 \mathrm{~g} \mathrm{~cm}^{-2}$.

A secagem de cada amostra $\left(1,5 \mathrm{~g} \mathrm{~cm}^{-2}\right)$ foi realizada em uma estufa com circulação forçada de ar (Nova Ética, modelo 400-5ND), nas temperaturas $40^{\circ} \mathrm{C}$, $50^{\circ} \mathrm{C}$ e $60^{\circ} \mathrm{C}$, sem interrupção da secagem até se atingir o equilíbrio. Durante a etapa de secagem, as bandejas ( $\phi: 30,5 \mathrm{~cm})$ contendo as amostras foram pesadas a cada 10 minutos até 70 minutos de secagem, sendo esse intervalo alterado para 30 minutos até 280 minutos de secagem, seguido de aumento do intervalo para 60 minutos até o término da secagem. A alteração do momento da mensuração das pesagens deve-se à diminuição da variação de massa em razão da secagem dos morangos, sendo desnecessária a mensuração a cada 10 minutos até o fim da secagem. A secagem foi finalizada quando a massa das bandejas com os frutos não variou mais de $0,01 \mathrm{~g}$ em três pesagens consecutivas, correspondendo a um conteúdo de umidade de 0,29 kg água/ $/ \mathrm{kg}$ de morango, 0,26 kg água/kg de morango e 0,21 kg água/kg de morango (b.u.), respectivamente, para as temperaturas $40^{\circ} \mathrm{C}, 50^{\circ} \mathrm{C}$ e $60^{\circ} \mathrm{C}$. Utilizou-se uma balança digital (Shimadzu, modelo BL3200H) com $0,01 \mathrm{~g}$ de resolução.

Para a determinação das razões do adimensional de umidade $(R U)$ dos morangos, durante a secagem, nas diferentes condições de temperatura do ar, foi utilizada a Equação 1:

$$
R U=\frac{U^{*}-U_{e}^{*}}{U_{i}^{*}-U_{e}^{*}}
$$

em que: RU é o adimensional de umidade; $U^{\star}$ é o teor de água do produto no tempo $\mathrm{t}(\mathrm{kg}$ água/kg de morango seco); $U^{*}$ é o teor de água de equilíbrio do produto; e $\mathrm{U}^{*}{ }_{\mathrm{i}}$ é o teor de água inicial do produto, quantificados em base seca.

Diferentes modelos propostos na literatura foram utilizados na previsão da secagem dos morangos (Tabela 1).

A energia de ativação é definida como energia que deve ser ultrapassada ou energia mínima requerida para iniciar uma reação química (OLIVEIRA et al., 2010). Dessa forma, foi calculada a energia de ativação na secagem dos morangos. A equação de Arrhenius (Equação 8), que relata a relação entre a energia de ativação e a velocidade em que a reação ocorre, foi utilizada.

Tabela 1. Modelos matemáticos utilizados para predizer o fenômeno de secagem.

\section{Nome do modelo}

Page

(OVERHULTS et al., 1973)

Logaritmo

(CHANDRA; SINGH, 1995)

Midilli modificado (GHAZANFARI et al., 2006)

Aproximação da Difusão (KASSEM, 1998)

Dois termos

(HENDERSON, 1974)

Verma

(VERMA et al., 1985)

\section{Modelo}

Equação

$$
\mathrm{RU}=\exp \left(-\mathrm{kt}^{\mathrm{c}}\right)
$$

$$
\mathrm{RU}=\mathrm{a} \exp (-\mathrm{kt})+\mathrm{b}
$$

$$
R U=\exp \left(-k t^{c}\right)+b t
$$

$$
R U=a \exp (-k t)+(1-a) \exp (-k b t)
$$

$$
R U=a \exp (-k t)+b \exp (-c t)
$$

$$
R U=a \exp (-k t)+(1-a) \exp (-b t)
$$

a, b, c são coeficientes dos modelos, adimensionais; $\mathrm{k}$ é a constante de secagem, $\mathrm{s}^{-1}$; t é o tempo, s. 


$$
k=A_{0} \exp \left(-\frac{E_{a}}{R T}\right)
$$

em que: $A_{0}$ é o fator pré-exponencial, $\mathrm{s}^{-1} ; \mathrm{E}_{\mathrm{a}}$ é a energia de ativação, $\mathrm{J} \mathrm{mol}^{-1}$; R é a constante universal dos gases, 8,314 $\mathrm{J} \mathrm{mol}^{-1} \mathrm{~K}^{-1}$; e T é a temperatura, $\mathrm{K}$.

Apesar de a constante de secagem $k$ ser apenas um ajuste de um modelo empírico, pode ser relacionada à equação de Arrhenius em razão de representar de modo satisfatório a cinética da secagem (KEEY, 1992; CHEN; MUJUMDAR, 2008). Ou seja, por relacionar a diminuição da razão de umidade com o tempo de secagem em diferentes temperaturas, essa constante pode ser relacionada às propriedades termodinâmicas do processo em estudo.

As propriedades termodinâmicas do processo de secagem foram obtidas por meio dos valores da constante de secagem obtidas pelo modelo que melhor se ajustou aos dados experimentais de secagem dos morangos. Dessa forma, os valores da variação de entalpia, entropia e energia livre de Gibbs foram calculados pelas Equações 9, 10 e 11, respectivamente (CORRÊA et al., 2012).

$$
\begin{aligned}
& \Delta H=E_{a}-R T \\
& \Delta S=R\left(\ln A_{0}-\ln \frac{k_{B}}{h_{P}}-\ln T\right) \\
& \Delta G=\Delta H-T \Delta S
\end{aligned}
$$

em que: $\Delta \mathrm{H}$ é variação de entalpia, $\mathrm{J} \mathrm{mol}^{-1} ; \Delta \mathrm{S}$ é a variação de entropia, $\mathrm{J} \mathrm{mol}^{-1} \mathrm{~K}^{-1} ; \Delta \mathrm{G}$ é a variação de energia livre de Gibbs, $\mathrm{J} \mathrm{mol}^{-1} ; \mathrm{k}_{\mathrm{B}}$ é a constante de Boltzmann, 1,38 × 10-23 $\mathrm{J} \mathrm{K}^{-1}$; e hp é a constante de Planck, $6,626 \times 10^{-34} \mathrm{~J} \mathrm{~s}$

Os dados experimentais da secagem dos morangos foram submetidos à análise de regressão e seleção do modelo matemático que melhor representou as curvas de secagem. Para o ajuste dos modelos matemáticos, foi realizada análise de regressão não linear pelo método Gauss Newton, utilizando-se o software STATISTICA 8.0 ${ }^{\circledR}$. A escolha do melhor modelo baseou-se nos seguintes parâmetros estatísticos: desvio-padrão da estimativa (SE) (Equação 12), erro médio relativo (P) (Equação 13), coeficiente de determinação $\left(R^{2}\right)$ e análise de resíduos.

$$
S E=\sqrt{\frac{\sum_{i=1}^{n}(Y-\hat{Y})^{2}}{G L R}}
$$

$P=\frac{100}{n} \sum_{i=1}^{n}\left(\frac{|Y-\hat{Y}|}{Y}\right)$

em que: SE é o desvio-padrão da estimativa, decimal; Y é o valor estimado experimentalmente; $\hat{Y}$ é o valor calculado pelo modelo; GLR são os graus de liberdade do modelo; $\mathrm{P}$ é o erro médio relativo, \%; e n é o número de dados observados.

\section{Resultados e discussão}

A Tabela 2 relata os valores de $\mathrm{P}, \mathrm{SE}$ e $\mathrm{R}^{2}$ para cada um dos modelos matemáticos utilizados nas diferentes temperaturas de secagem dos morangos.

Para que um modelo seja adequado na descrição de um fenômeno, segundo Mohapatra e Rao (2005), valores inferiores a $10 \%$ de erro médio relativo $(P)$ indicam bom ajuste para fins práticos. De acordo com Draper e Smith (1998), a capacidade de um modelo para descrever com fidelidade determinado processo físico é inversamente proporcional ao valor do erro-padrão da estimativa (SE). Entretanto, o coeficiente de determinação $\left(R^{2}\right)$ para modelos não lineares não é uma boa ferramenta de tomada de decisão, sendo necessária a análise conjunta dos três parâmetros estatísticos.

Tendo em vista esses parâmetros estatísticos, verificou-se, pela Tabela 2, que os modelos aptos a descrever a secagem na temperatura de $40^{\circ} \mathrm{C}$ foram o da Aproximação da Difusão e o Logaritmo; na temperatura de $50^{\circ} \mathrm{C}$, os modelos de Aproximação da Difusão, Logaritmo, de Midilli modificado e Verma; e para a secagem a $60^{\circ} \mathrm{C}$, os modelos de Logaritmo, de Midilli modificado e Verma.

\begin{tabular}{|c|c|c|c|}
\hline \multicolumn{4}{|c|}{ Temperatura $40{ }^{\circ} \mathrm{C}$} \\
\hline $\begin{array}{c}\text { Designação do } \\
\text { modelo }\end{array}$ & $\mathbf{P}(\%)$ & $\begin{array}{c}\text { SE } \\
\text { (decimal) }\end{array}$ & $\mathbf{R}^{2}(\%)$ \\
\hline $\begin{array}{l}\text { Aproximação da } \\
\text { difusão }\end{array}$ & 8,60 & 0,14 & 99,56 \\
\hline Dois Termos & 21,99 & 0,12 & 99,67 \\
\hline Logaritmo & 8,09 & 0,06 & 99,93 \\
\hline Midilli modificado & 14,83 & 0,11 & 99,74 \\
\hline Page & 30,51 & 0,20 & 99,08 \\
\hline Verma & 27,01 & 0,19 & 99,22 \\
\hline \multicolumn{4}{|l|}{ Temperatura $50^{\circ} \mathrm{C}$} \\
\hline $\begin{array}{l}\text { Aproximação da } \\
\text { difusão }\end{array}$ & 6,66 & 0,04 & 99,93 \\
\hline Dois Termos & 59,74 & 1,05 & 62,84 \\
\hline Logaritmo & 8,48 & 0,04 & 99,93 \\
\hline Midilli modificado & 8,99 & 0,05 & 99,91 \\
\hline Page & 19,44 & 0,11 & 99,56 \\
\hline Verma & 6,66 & 0,04 & 99,93 \\
\hline \multicolumn{4}{|l|}{ Temperatura $60^{\circ} \mathrm{C}$} \\
\hline $\begin{array}{l}\text { Aproximação da } \\
\text { difusão }\end{array}$ & 19,38 & 0,08 & 99,70 \\
\hline Dois Termos & 17,80 & 0,07 & 99,76 \\
\hline Logaritmo & 7,59 & 0,03 & 99,95 \\
\hline Midilli modificado & 8,33 & 0,04 & 99,93 \\
\hline Page & 20,51 & 0,09 & 99,62 \\
\hline Verma & 5,55 & 0,03 & 99,95 \\
\hline
\end{tabular}
Entretanto, a modelagem tem como objetivo adequar

Tabela 2. Valores de erro médio relativo $(P)$, desvio-padrão da estimativa (SE) e coeficiente de determinação $\left(R^{2}\right)$ para modelos matemáticos de secagem dos morangos. 
um único modelo em toda faixa estudada da variável em questão (adimensional de umidade), nas diferentes temperaturas utilizadas. Devido a esse fato, o modelo Logaritmo foi considerado adequado para descrever a etapa de secagem nas temperaturas $40^{\circ} \mathrm{C}, 50^{\circ} \mathrm{C}$ e $60^{\circ} \mathrm{C}$. Esse modelo possibilitou a obtenção de valores de SE de no máximo 0,06 e de $\mathrm{R}^{2}$ entre 99,93\% e 99,95\%. Por sua vez, Sousa et al. (2014) concluíram que a secagem de morangos cortados longitudinalmente, nas temperaturas $40{ }^{\circ} \mathrm{C}, 55^{\circ} \mathrm{C}$ e $70^{\circ} \mathrm{C}$, pode ser descrita pelo modelo de Midilli modificado. Essa diferença indica a necessidade de estudo de um mesmo produto em diferentes formas e em diferentes temperaturas de secagem.

A Figura 1 mostra o comportamento do adimensional de umidade com o tempo de secagem dos morangos nas três diferentes condições utilizadas, bem como os valores estimados para a secagem pelo modelo Logaritmo.

Pode-se observar que o adimensional de umidade da secagem de morangos em menor temperatura levou mais tempo para chegar ao equilíbrio comparado ao tempo da secagem em temperatura maior. Esse fato pode ser explicado pela maior pressão de vapor interna dos morangos, acarretada pela maior temperatura de secagem, levando à maior saída de moléculas de água do produto, uma vez que permite que o ar absorva maior quantidade de umidade.

Pela Figura 1 nota-se que o modelo Logaritmo, ao final da secagem, aumentou seu erro, subestimando os valores da razão de umidade nesse período. Esse fato é esperado, uma vez que os modelos refletem principalmente a parte exponencial (decrescente) da secagem, tornando-se uma limitação no final desse processo, uma vez que se tende à condição de equilíbrio, chegando a valores constantes, para os quais a previsão do modelo apresentou pouca sensibilidade. Esse erro aumentou com o incremento da temperatura de secagem.

A Tabela 3 apresenta as Equações 14, 15 e 16 do modelo Logaritmo com inclusão das constantes obtidas na modelagem, para representação da secagem dos morangos.

A constante de secagem $(k)$ é uma aproximação do efeito da temperatura na secagem, relacionando-se à difusividade efetiva no processo de secagem no período decrescente e à difusão líquida que controla o processo (MADAMBA et al., 1996; BABALIS; BELESSIOTIS, 2004). Pode-se observar, pelas Equações 14, 15 e 16 na

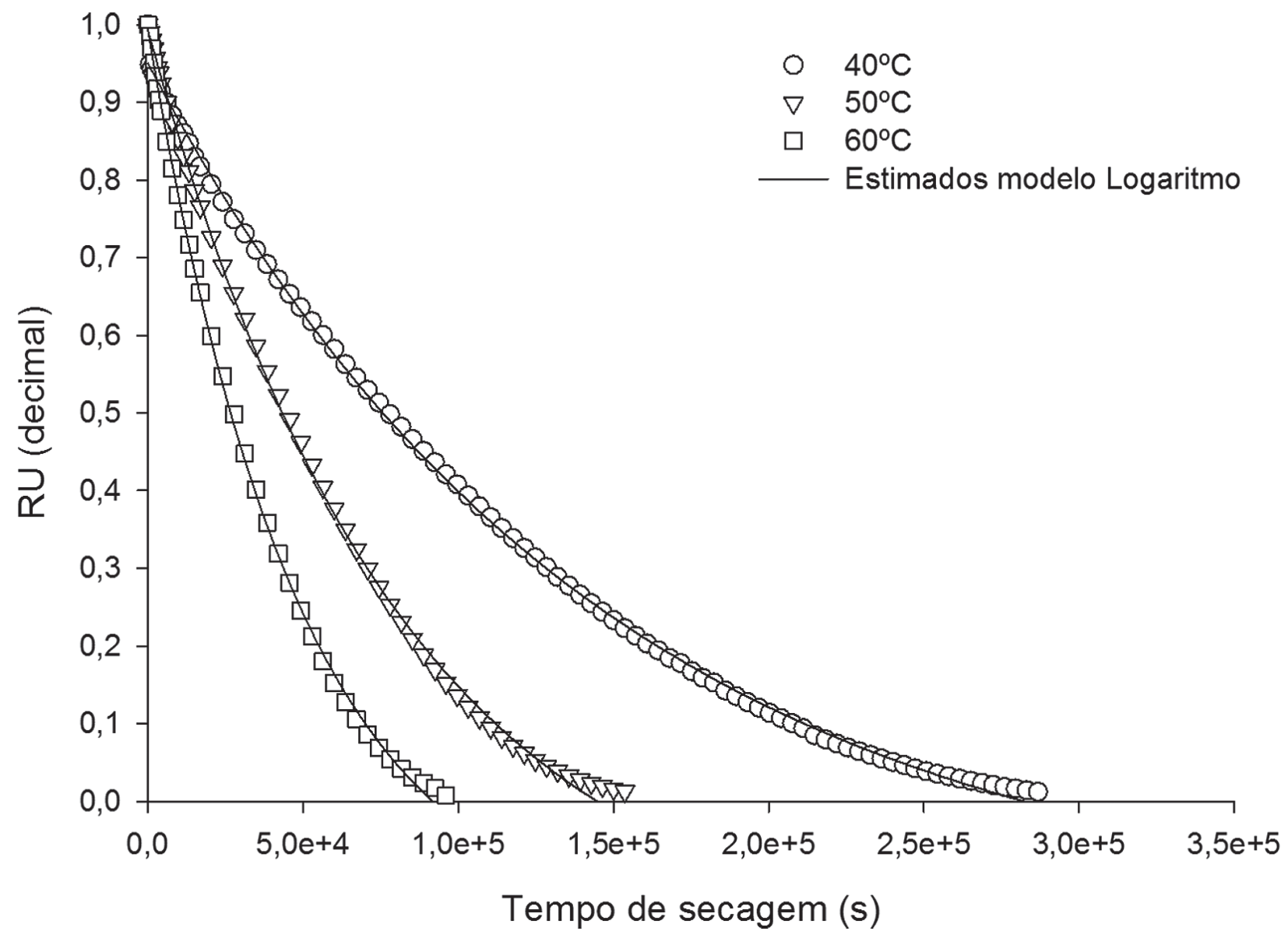

Figura 1. Dados observados e estimados pelo modelo Logaritmo na secagem de morangos. 
Tabela 3. Modelo Logaritmo ajustado aos dados experimentais de secagem de morangos.

\begin{tabular}{ccc} 
Temperatura $\left({ }^{\circ} \mathbf{C}\right)$ & Logaritmo & Equação \\
40 & $\mathrm{RU}=1,1093 \times \exp \left(-0,7 \times 10^{-5} \mathrm{t}\right)-0,1592$ \\
50 & $\mathrm{RU}=1,1950 \times \exp \left(-1,2 \times 10^{-5} \mathrm{t}\right)-0,2022$ \\
60 & $\mathrm{RU}=1,1727 \times \exp \left(-2,1 \times 10^{-5} \mathrm{t}\right)-0,1792$ \\
\hline
\end{tabular}

Tabela 3, que a constante de secagem $\mathrm{k}$ cresceu, em valores absolutos, com o aumento da temperatura, variando de 0,7 a $2,1 \times 10^{-5} \mathrm{~s}^{-1}$

A energia de ativação aferida para a obtenção do teor de água de equilíbrio foi de 2.122,40 $\mathrm{J} \mathrm{mol}^{-1}$. Segundo Corrêa et al. (2010), nos processos de secagem, quanto menor for a energia de ativação maior será a difusividade de água no produto. Ou seja, menor será a energia necessária para que a transformação física ocorra, nesse caso, a transformação da água livre líquida em vapor (secagem). Esses autores encontraram um valor de 38.389,90 $\mathrm{J} \mathrm{mol}^{-1}$, indicando que a secagem de morangos se dá com maior facilidade que a secagem de frutos de café.

Os valores de variação de entalpia foram inversamente proporcionais aos de temperatura, $-481,21 \mathrm{~J} \mathrm{~mol}^{-1},-564,35 \mathrm{~J} \mathrm{~mol}^{-1}$ e $-647,49 \mathrm{~J} \mathrm{~mol}^{-1}$, respectivamente, para as temperaturas $40{ }^{\circ} \mathrm{C}, 50{ }^{\circ} \mathrm{C}$ e $60{ }^{\circ} \mathrm{C}$. Os valores negativos de variação de entalpia diferencial representam uma transformação exotérmica, ou seja, processo com liberação de calor. Ascheri et al. (2009) encontraram valores entre $-6.990,00$ e 21.000,00 $\mathrm{J} \mathrm{mol}^{-1}$ para o processo de adsorção de água em rizomas de lírio-do-brejo. Rodríguez-Bernal et al. (2015) encontraram valores de variação de entalpia entre -30.000,00 e -57.416,00 $\mathrm{J} \mathrm{mol}^{-1}$ para a adsorção de água pela polpa de borojó. Já para a secagem de frutos de café cereja, Corrêa et al. (2010) reportaram valores de variação de entalpia de 35.827,85 $\mathrm{J} \mathrm{mol}^{-1}, 35.744,71 \mathrm{~J} \mathrm{~mol}^{-1} \mathrm{e}$ $35.661,57 \mathrm{~J} \mathrm{~mol}^{-1}$ para as temperaturas $35^{\circ} \mathrm{C}, 45^{\circ} \mathrm{C}$ e $55^{\circ} \mathrm{C}$, respectivamente. Menores valores de variação de entalpia indicam menor energia necessária para remover a água ligada ao produto durante a secagem (OLIVEIRA et al., 2010), sendo assim, nota-se que a secagem de morangos ocorreu mais facilmente em relação à secagem de café e a outros processos, uma vez que os valores de entalpia encontrados no presente trabalho são significativamente inferiores aos dos demais trabalhos.

No caso do processo de secagem descrito, os valores de variação de entalpia permitiram concluir que há uma maior estabilidade das interações das moléculas de água, entre si, e das moléculas do produto, entre si, em comparação àquelas entre a água e o produto, ou seja, as interações água-água e produto-produto são entalpicamente mais estáveis em comparação às interações água-produto, repercutindo em uma diminuição de entalpia ao final de secagem.

Ao mesmo tempo, o aumento da variação da entalpia do processo de secagem dos morangos com o aumento da temperatura já era esperado, uma vez que valores elevados de temperatura facilitaram o processo de secagem por diminuírem a energia necessária para remover moléculas de água ligadas ao produto.

Para a entropia, os valores encontrados foram $-238,35 \mathrm{~J} \mathrm{~mol}^{-1} \mathrm{~K}^{-1},-238,61 \mathrm{~J} \mathrm{~mol}^{-1} \mathrm{~K}^{-1} \mathrm{e}-238,86 \mathrm{~J} \mathrm{~mol}^{-1} \mathrm{~K}^{-1}$ para as temperaturas $40^{\circ} \mathrm{C}, 50^{\circ} \mathrm{C}$ e $60^{\circ} \mathrm{C}$, respectivamente. A pequena variação deveu-se à metodologia utilizada, que se baseia nos números de Planck e de Boltzmann (constantes), sendo a variação de entropia apenas retratada pela variação de temperatura, pois os demais parâmetros são constantes. Como a variação da temperatura é baixa $\left(10^{\circ} \mathrm{C}\right)$, os logaritmos naturais das temperaturas são muito próximos, relatando essa variação mínima na entropia. Corrêa et al. (2010) reportaram valores de entropia de $-142,20 \mathrm{~J} \mathrm{~mol}^{-1} \mathrm{~K}^{-1},-142,46 \mathrm{~J} \mathrm{~mol}^{-1} \mathrm{~K}^{-1}$ e $-142,72 \mathrm{~J} \mathrm{~mol}^{-1} \mathrm{~K}^{-1}$ para as temperaturas $35^{\circ} \mathrm{C}, 45^{\circ} \mathrm{C}$ e $55^{\circ} \mathrm{C}$, respectivamente. Rodríguez-Bernal et al. (2015) encontraram valores de entropia entre -150,00 e -30,00 $\mathrm{J} \mathrm{mol}^{-1} \mathrm{~K}^{-1}$ para a adsorção de água pela polpa de borojó. Os valores negativos de entropia foram atribuídos à existência de adsorção química e/ou modificações estruturais do adsorvente (MOREIRA et al., 2008).

A energia livre de Gibbs busca medir a totalidade de energia associada a um sistema termodinâmico e o seu valor positivo é explicado por uma adição de energia que envolve o produto para a ocorrência da mudança de fase (líquido para vapor). No caso de secagem não existe reação química, o que torna essa operação não espontânea nessas condições. Os valores da energia livre de Gibbs foram 74.159,75 $\mathrm{J} \mathrm{mol}^{-1}$, $76.544,54 \mathrm{~J} \mathrm{~mol}^{-1}$ e $78.931,90 \mathrm{~J} \mathrm{~mol}^{-1}$ para $40{ }^{\circ} \mathrm{C}, 50^{\circ} \mathrm{C}$ e $60{ }^{\circ} \mathrm{C}$, respectivamente, os quais demonstraram a não espontaneidade do processo. Trabalhos anteriores relataram valores e tendência similares aos apresentados no presente trabalho, como para a secagem de café cereja: $79.646,81 \mathrm{~J} \mathrm{~mol}^{-1}, 81.070,10 \mathrm{~J} \mathrm{~mol}^{-1} \mathrm{e}$ 82.496,01 $\mathrm{J} \mathrm{mol}^{-1}$ para as temperaturas $35^{\circ} \mathrm{C}, 45^{\circ} \mathrm{C}$ 
e $55^{\circ} \mathrm{C}$, respectivamente (CORRÊA et al., 2010); e para a secagem de folhas de timbó: 140.108,1 $\mathrm{J} \mathrm{mol}^{-1}$, 142.067,6 $\mathrm{J} \mathrm{mol}^{-1}, 144.029,7 \mathrm{~J} \mathrm{~mol}^{-1}$ e 145.994,2 $\mathrm{J} \mathrm{mol}^{-1}$ para as temperaturas $40{ }^{\circ} \mathrm{C}, 50^{\circ} \mathrm{C}, 60^{\circ} \mathrm{C}$ e $70{ }^{\circ} \mathrm{C}$, respectivamente (MARTINS et al., 2015).

A não espontaneidade do processo de secagem descrito corrobora os valores de entalpia e entropia mostrados anteriormente, pois, de acordo com a Equação 11, processos não espontâneos ocorrem em três situações distintas: em que a variação de entropia é positiva e a variação de entropia é negativa; em que as variações de entalpia e entropia são positivas, mas o valor de $\Delta \mathrm{H}$ é maior que $\mathrm{T} \Delta \mathrm{S}$; ou em que a entalpia e a entropia são negativas mas o valor de $\Delta \mathrm{H}$ é menor que $\mathrm{T} \Delta \mathrm{S}$, que é o caso deste trabalho.

\section{Conclusões}

Para o presente estudo da modelagem e obtenção dos parâmetros termodinâmicos durante o processo de secagem de morangos (Fragaria L.) em três diferentes condições de temperatura $\left(40^{\circ} \mathrm{C}, 50^{\circ} \mathrm{C}\right.$ e $\left.60^{\circ} \mathrm{C}\right)$ pode-se concluir que:

- A temperatura do ar de secagem que proporcionou menor tempo de secagem foi a de $60{ }^{\circ} \mathrm{C}$, com uma constante de secagem de $2,1 \times 10^{-5} \mathrm{~s}^{-1}$

- Na modelagem matemática, baseando-se nos parâmetros estatísticos e na análise da aplicabilidade prática, o modelo escolhido, com ajuste satisfatório em toda a faixa de temperaturas de secagem utilizadas, foi o modelo Logaritmo;

- Os valores de entalpia e entropia decrescem com o aumento da temperatura, sendo ambas negativas em todas as condições de secagem do presente trabalho;

- A energia livre de Gibbs é diretamente proporcional à temperatura, sendo a secagem não espontânea, requerendo-se a inserção de energia para que o processo ocorra.

\section{Agradecimentos}

Os autores agradecem o Conselho Nacional de Desenvolvimento Científico e Tecnológico (CNPq) pelo indispensável auxílio na forma de bolsa de iniciação científica.

\section{Referências}

ANTUNES, L. E. C.; REISSER JUNIOR, C. Produção de morangos. Jornal da Fruta, Lages, v. 15, n. 191, p. 22-24, 2007.
ASCHERI, D. P. R.; MOURA, W. S.; ASCHERI, J. L. R.; FREITAS JUNIOR, E. A. Propriedades termodinâmicas de adsorção de água do amido de rizomas do lírio-do-brejo (Hedychium coronarium). Ciência e Tecnologia de Alimentos, Campinas, v. 29, n. 2, p. 454-462, 2009. http://dx.doi.org/10.1590/S010120612009000200036.

BABALIS, S. J.; BELESSIOTIS, V. G. Influence of the drying conditions on the drying constants and moisture diffusivity during the thin-layer drying of figs. Journal of Food Engineering, Essex, v. 65, n. 3, p. 449-458, 2004. http://dx.doi.org/10.1016/j. jfoodeng.2004.02.005.

BERBERT, P. A.; QUEIROZ, D. M.; SILVA, J. S.; PINHEIRO FILHO, J. B. Simulation of coffee drying in a fixed bed with periodic airflow reversal. Journal of Agricultural Engineering Research, v. 60, n. 3, p. 167-173, 1995. http://dx.doi.org/10.1006/ jaer.1995.1010.

BRASIL. Ministério da Agricultura e Reforma Agrária. Regras para análise de sementes. Brasília: SNDA; DNDV; CLAV, 1992. $365 \mathrm{p}$.

CHANDRA, P. K.; SINGH, R. P. Applied numerical methods for food and agricultural engineers. Boca Raton: CRC Press, 1995. $512 \mathrm{p}$.

CHEN, X. D.; MUJUMDAR, A. S. Drying technologies in food processing. United Kingdom: John Wiley \& Sons Ltda., 2008. $322 \mathrm{p}$.

CONTRERAS, C.; MARTÍN-ESPARZA, M. E.; CHIRALT, A.; MARTÍNEZ-NAVARRETE, N. Influence of microwave application on convective drying: effects on drying kinetics, and optical and mechanical properties of apple and strawberry. Journal of Food Engineering, Essex, v. 88, n. 1, p. 55-64, 2008. http://dx.doi. org/10.1016/j.jfoodeng.2008.01.014.

CORRÊA, P. C.; OLIVEIRA, G. H. H.; BOTELHO, F. M.; GONELI, A. L. D.; CARVALHO, F. M. Modelagem matemática e determinação das propriedades termodinâmicas do café (Coffea arabica L.) durante o processo de secagem. Revista Ceres, Viçosa, v. 57, n. 5, p. 595-601, 2010. http://dx.doi.org/10.1590/S0034$737 \times 2010000500005$.

CORRÊA, P. C.; OliveiRA, G. H. H.; SANTOS, E. S. Thermodynamic properties of agricultural products processes. In: ARANA, I. (Org.). Physical properties of foods: novel measurement techniques and applications. Boca Raton: CRC Press, 2012. p. 131-141.

DRAPER, N. R.; SMITH, H. Applied regression analysis. New York: John Wiley \& Sons, 1998. 736 p.

EL-BELTAGY, A.; GAMEA, G. R.; AMER ESSA, A. H. Solar drying characteristics of strawberry. Journal of Food Engineering, v. 78, n. 2, p. 456-464, 2007. http://dx.doi.org/10.1016/j. jfoodeng.2005.10.015.

EMPRESA DE ASSISTÊNCIA TÉCNICA E EXTENSÃO RURAL - EMATER. Informações agropecuárias do Distrito Federal - 
Modelagem e propriedades termodinâmicas na secagem de morangos

OLIVEIRA, G. H. H. et al.

2013. Curitiba, 2013. Disponível em: <http://www.emater.df.gov. br/index.php?option=com_phocadownload \&view=category $\&$ do wnload=863: informaoes-agropecuarias-2013\&id=72: arquivosrelacionados-relatorios-e-informacoes-agropecuarias\&ltemid=55/>. Acesso em: 19 dez. 2015.

GHAZANFARI, A.; EMAMI, S.; TABIL, L. G.; PANIGRAHI, S. Thin-layer drying of flax fiber: II. Modeling drying process using semi-theoretical and empirical models. Drying Technology, New York, v. 24, n. 12, p. 1637-1642, 2006. http://dx.doi. org/10.1080/07373930601031463

HENDERSON, S. M. Progress in developing the thin-layer drying equation. Transactions of the ASAE. American Society of Agricultural, St. Joseph, v. 17, n. 6, p. 1167-1168, 1974. http:// dx.doi.org/10.13031/2013.37052

KASSEM, A. S. Comparative studies on thin layer drying models for wheat. In: INTERNATIONAL CONGRESS ON AGRICULTURAL ENGINEERING, 13., 1998, Rabat. Proceedings... Rabat: ANAFID, 1998, v. 6, p. 2-6

KEEY, R. B. Drying of loose and particulate materials. New York: Hemisphere Publishing Coroporation, 1992. 505 p.

MADAMBA, P. S.; DRISCOLL, R. H.; BUCKLE, K. A. Thinlayer drying characteristics of garlic slices. Journal of Food Engineering, Essex, v. 29, n. 1, p. 75-97, 1996. http://dx.doi. org/10.1016/0260-8774(95)00062-3

MARTINS, E. A. S.; LAGE, E. Z.; GONELI, A. L. D.; HARTMANN FILHO, C. P.; LOPES, J. G. Cinética de secagem de folhas de timbó (Serjania marginata Casar). Revista Brasileira de Engenharia Agrícola e Ambiental, Campina Grande, v. 19, n. 3, p. 238-244, 2015.

MOHAPATRA, D.; RAO, P. S. A thin layer drying model of parboiled wheat. Journal of Food Engineering, California, v. 66, n. 4, p. 513-518, 2005. http://dx.doi.org/10.1016/j. jfoodeng.2004.04.023.

MOREIRA, R.; CHENLO, F.; TORRES, M. D.; VALLEJO, N. Thermodynamic analysis of experimental sorption isotherms of loquat and quince fruits. Journal of Food Engineering, California, v. 88, n. 4, p. 514-521, 2008. http://dx.doi. org/10.1016/j.jfoodeng.2008.03.011

MORENO, J.; SIMPSON, R.; BAEZA, A.; MORALES, J.; MUÑOZ, C.; SATRY, S.; ALMONACID, S. Effect of ohmic heating and vacuum impregnation on the osmodehydration kinetics and microstructure of strawberries (cv. Camarosa). LWT - Food Science and Technology, Zurique, v. 45, p. 148-154, 2012.

MOSQUERA, L. H.; MORAGA, G.; MARTÍNEZ-NAVARRETE, N. Critical water activity and critical water content of freeze-dried strawberry powder as affected by maltodextrin and arabic gum. Food Research International, Campinas, v. 47, n. 2, p. 201-206, 2012. http://dx.doi.org/10.1016/j.foodres.2011.05.019.

NUÑEZ-MANCILLA, Y.; PÉREZ-WON, M.; URIBE, E.; VEGAGÁLVEZ, A.; DI SCALA, K. Osmotic dehydration under high hydrostatic pressure: effects on antioxidant activity, total phenolics compounds, vitamin C and colour of strawberry (Fragaria vesca). LWT - Food Science and Technology, Zurique, v. 52, p. 151-156, 2013

NUÑEZ-MANCILLA, Y.; PEREZ-WON, M.; VEGA-GÁLVEZ, A.; ARIAS, V.; TABILO-MUNIZAGA, G.; BRIONES-LABARCA, V.; LEMUS-MONDACA, R.; DI SCALA, K. Modeling mass transfer during osmotic dehydration of strawberries under high hydrostatic pressure conditions. Innovative Food Science and Emerging Technologies, Berlim, v. 12, n. 3, p. 338-343, 2011. http://dx.doi.org/10.1016/j.ifset.2011.03.005.

OLIVEIRA, G. H. H.; CORRÊA, P. C.; ARAÚJO, E. F.; VALENTE, D. S. M.; BOTELHO, F. M. Desorption isotherms and thermodynamic properties of sweet corn cultivars (Zea mays L.). International Journal of Food Science and Technology, Londres, v. 45, n. 3, p. 546-554, 2010. http://dx.doi.org/10.1111/j.13652621.2009.02163.x

OLIVEIRA, G. H. H.; CORRÊA, P. C.; SANTOS, E. S.; TRETO, P. C.; DINIZ, M. D. M. S. Evaluation of thermodynamic properties using GAB model to describe the desorption process of cocoa beans. International Journal of Food Science and Technology, Londres, v. 46, n. 10, p. 2077-2084, 2011. http:// dx.doi.org/10.1111/j.1365-2621.2011.02719.x.

OVERHULTS, D. D.; WHITE, G. M.; HAMILTON, M. E.; ROSS, I. J. Drying soybeans with heated air. Transactions of the ASAE. American Society of Agricultural Engineers, St. Joseph, v. 16, n. 2, p. 195-200, 1973.

REISSER JUNIOR, C.; ANTUNES, L. E. C.; RADIN, B. Produção de morango. In: SIMPÓSIO DO MORANGO, 5., ENCONTRO SOBRE PEQUENAS FRUTAS E FRUTAS NATIVAS DO MERCOSUL, 4., 2010, Pelotas. Anais... Pelotas: Embrapa Clima Temperado, 2010. $216 \mathrm{p}$.

RESENDE, L. M. A.; MASCARENHAS, M. H. T.; PAIVA, B. M. Panorama da produção e comercialização do morango. Informe Agropecuário, Belo Horizonte, v. 20, n. 198, p. 5-19, 1999.

RODRÍGUEZ-BERNAL, J. M.; FLORES-ANDRADE, E.; LIZARAZO-MORALES, C.; BONILLA, E.; PASCUAL-PINEDA, L. A.; GUTIERRÉZ-LÓPEZ, G.; QUINTANILLA-CARVAJAL, M. $X$. Moisture adsorption isotherms of the borojó fruit (Borojoa patinoi. Cuatrecasas) and gum arabic powders. Food and Bioproducts Processing, Rugby, v. 94, p. 187-198, 2015. http:// dx.doi.org/10.1016/j.fbp.2015.03.004.

SOUSA, F. A.; ANDRADE, E. T.; FIGUEIRA, V. G.; D'ANDREA, E. M.; CORREAA FILHO, L. C. Estudo da cinética de secagem, contração volumétrica e análise da difusão líquida do morango (Fragaria sp.). Engevista, Niterói, v. 16, n. 1, p. 8-18, 2014

VERMA, L. R.; BUCKLIN, R. A.; ENDAN, J. B.; WRATTEN, F. T. Effects of drying air parameters on rice drying models. Transactions of the ASAE. American Society of Agricultural Engineers, St. Joseph, v. 28, n. 1, p. 296-301, 1985. http://dx.doi. org/10.13031/2013.32245. 